\title{
Computed Tomography with Contrast
}

National Cancer Institute

\section{Source}

National Cancer Institute. Computed Tomography with Contrast. NCI Thesaurus. Code C137805.

Computed tomography that uses a contrast agent to visualize anatomic structures. 